\title{
Cumulative Probability of Prostate Cancer Detection Using the International Prostate Symptom Score in a Prostate-specific Antigen-based Population Screening Program in Japan
}

\author{
Yasuhide Kitagawa*, Satoko Urata, Kazutaka Narimoto, Tomomi Nakagawa, \\ Kouji Izumi, Yoshifumi Kadono, Hiroyuki Konaka, Atsushi Mizokami, Mikio \\ Namiki
}

\begin{abstract}
The International Prostate Symptom Score (IPSS) is often used as an interview sheet for assessing lower urinary tract symptoms (LUTS) at the time of prostate-specific antigen (PSA) testing during population-based screening for prostate cancer. However, the relationship between prostate cancer detection and LUTS status remains controversial. To elucidate this relationship, the cumulative probability of prostate cancer detection using IPSS in biopsy samples from patients categorized by serum PSA levels was investigated. The clinical characteristics of prostate cancer detected using IPSS during screening were also investigated. A total of 1,739 men aged 54-75 years with elevated serum PSA levels who completed the IPSS questionnaire during the initial population screening in Kanazawa City, Japan and underwent systematic transrectal ultrasonography-guided prostate biopsy between 2000 and 2013 were enrolled in the present study. Of the 1,739 men, $544(31.3 \%)$ were diagnosed with prostate cancer during the observation period. The probability of cancer detection at 3 years in the entire study population was $27.4 \%$ and $32.7 \%$ for men with IPSS $\leq 7$ and those with IPSS $\geq 8$, respectively; there was no statistically significant difference between groups. In men with serum PSA levels of 6.1 to 12.0ng/ $\mathrm{mL}$ at initial screening, the probability of cancer detection was significantly higher in men with IPSS $\leq 7$ than in those with IPSS $\geq 8$. There were no significant differences in clinical characteristics between groups of patients stratified by IPSS. These findings indicate that the use of IPSS for LUTS status evaluation may be useful for prostate cancer detection in the limited range of serum PSA levels.
\end{abstract}

Keywords: Prostate cancer - International Prostate Symptom Score - screening - risk factor

Asian Pac J Cancer Prev, 15 (17), 7079-7083

\section{Introduction}

Prostate-specific antigen (PSA)-based screening is widely used for the early detection of prostate cancer. The high rate of PSA testing among middle-aged men may have partially contributed to the continuous decrease in prostate cancer mortality in several countries (Bouchardy et al., 2008). In Asian countries, the screening rate for prostate cancer is still very low compared with that in the USA and West Europe. Consequently, it is predicted that the incidence and mortality rates for prostate cancer will increase in the future (Ito et al., 2009; Kash et al., 2014; Shin et al., 2014). Since the 1990s, each municipal government in Japan has provided prostate cancer screening systems, and a decreased mortality rate for prostate cancer is expected in some regions with a marked increase in the PSA testing rate (Ito et al., 2009). PSA-based screening systems implemented by municipal governments are reasonable measures to increase the exposure to PSA testing; several studies have already suggested that these systems may be effective for the early detection of prostate cancer (Kubota et al., 2002; Okihara et al., 2008; Kitagawa et al., 2011).

During the screening program, lower urinary tract symptoms (LUTS) of participants are often assessed using the International Prostate Symptom Score (IPSS) questionnaire at the time of PSA testing for the early detection of all types of illnesses (Matsubara et al., 2006). However, the relationship between prostate cancer detection and LUTS status in a population-based screening cohort remains controversial (Matsubara et al., 2006; Franlund et al., 2012).

In this study, we examined the relationship between prostate cancer detection and IPSS in a PSA-based population screening cohort and investigated the cumulative probability of cancer detection in prostate biopsy samples using IPSS during an initial population screening. The clinical characteristics of prostate cancer 
detected using IPSS during population screening were also investigated.

\section{Materials and Methods}

Since 2000, PSA-based annual population screening for prostate cancer has been provided for men aged 5469 years in Kanazawa City, Japan (Kobori et al., 2008; Kitagawa et al., 2011). Serum total PSA (tPSA) levels were measured in all participants using a Tosoh II PA kit (Tosoh Corporation, Tokyo, Japan) as the primary screening modality, and all participants were asked to complete the IPSS questionnaire as an interview sheet for LUTS at the time of PSA testing. Participants with serum tPSA levels of $\leq 2.0 \mathrm{ng} / \mathrm{mL}$ did not proceed to additional examinations and were recommended to undergo annual screening. Serum free PSA (fPSA) levels were measured in participants with tPSA levels within the range of 2.1$10.0 \mathrm{ng} / \mathrm{mL}$ using an Immulyze Free PSA kit (Nippon DPC Co. Ltd., Chiba, Japan). From 2000 to 2002, participants with serum tPSA levels $>2.0 \mathrm{ng} / \mathrm{mL}$ were recommended to proceed to further examination by urologists at primary medical care clinics. After 2003, participants with serum tPSA $>10.0 \mathrm{ng} / \mathrm{mL}$ and those with an $\mathrm{f} / \mathrm{t}$ PSA ratio of $\leq 0.22$ within the PSA reflex range of $2.1-10.0 \mathrm{ng} / \mathrm{mL}$ were recommended to proceed to closer examination (Kobori et al., 2008; Kitagawa et al., 2011). Since 2012, the age range of participants has increased to 54-75 years. Men with IPSS $\geq 8$ and serum PSA levels below the threshold value were followed by their general practitioners and were recommended to consult a urology clinic if they had urological complaints.

PSA levels were measured again in all men who proceeded to closer examination and underwent both digital rectal examination (DRE) and transrectal ultrasonography (TRUS). Systematic TRUS-guided prostate biopsy (6-12 cores) was recommended for men with any abnormal findings on repeat PSA assessment, DRE, or TRUS. If individuals refused to undergo prostate biopsy or if the urologists did not recommended biopsy, patients were followed up by annual PSA testing at subsequent population-based screenings.

In patients diagnosed with prostate cancer, tumors were pathologically graded by local pathologists, and, on the basis of DRE, TRUS, computed tomography, magnetic resonance imaging, and bone scanning findings at each urology department, clinical staging was determined according to the Union for International Cancer Control tumor, node, metastasis classification published in 1997 (International Union Against Cancer 1997). Medical information, including PSA levels at the primary screening, biopsy results, and clinicopathological findings, was reported to the office of Kanazawa Medical Association.

In the Kanazawa population-based screening cohort, 22,252 men participated in the screening program during the 14 years from 2000 through 2013, and 1,758 (7.9\%) underwent prostate biopsy. Of these 1,758 men, 1,739 $(98.9 \%)$ with available IPSS at the initial population screening were enrolled into the present study. IPSS was used to indicate the presence of LUTS: men with absent or mild LUTS (IPSS $\leq 7$ ) and moderate or severe LUTS (IPSS $\geq 8$ ). We investigated the cumulative probability of cancer detection using IPSS in prostate biopsy samples from patients categorized by serum PSA levels. The time to detection of prostate cancer by prostate biopsy was calculated from the date of initial population screening, and participants without evidence of cancer were censored at the date of their last prostate biopsy.

The principles of the Declaration of Helsinki were followed in this retrospective analysis. Comparisons between the two groups were performed by the MannWhitney U test or Fisher's exact test. The probability of prostate cancer detection using IPSS were examined by Kaplan-Meier analysis, and the significance of differences was analyzed by the log-rank test. All statistical analyses were performed using commercially available software (SPSS Statistics; IBM Corporation, Armonk, New York), and figures were prepared using Prism GraphPad Software (San Diego, California). $\mathrm{p}<0.05$ was considered statistically significant.

\section{Results}

The characteristics of 1,739 men eligible for this study are shown in Table 1 . Of the 1,739 men who underwent systematic TRUS-guided prostate biopsy, $544(31.3 \%)$ were diagnosed with prostate cancer during the observation period. In terms of prostate biopsy-based diagnosis, there were no statistically significant differences in the duration between screening and diagnosis and the number of prostate biopsies. Age and serum PSA levels at initial screening were significantly higher in men with prostate cancer than in those without. In terms of IPSS, 625 (35.9\%) and 110 (6.3\%) men had moderate (IPSS, 8-19) and severe LUTS (IPSS, 20-35), respectively. There was no statistically significant difference in IPSS; however, the proportion of men with IPSS $\geq 20$ was significantly lower among men with prostate cancer than among those without.

Table 2 shows the characteristics of participants stratified by LUTS using IPSS. Age and serum PSA levels at initial screening were significantly higher in men with moderate or severe LUTS (IPSS $\geq 8$ ) than in those with absent or mild LUTS (IPSS $\leq 7$ ). The proportion of men with serum PSA $\leq 2.0 \mathrm{ng} / \mathrm{mL}$ was significantly higher among those with absent or mild LUTS (IPSS $\leq 7$ ) than among those with moderate or severe LUTS (IPSS $\geq 8$ ). There was no statistically significant difference in the detection rate of prostate cancer between groups.

Kaplan-Meier curves for the cumulative probability of prostate cancer detection using IPSS are shown in Figure 1 . The probability of prostate cancer detection at 3 years in the entire study population was $27.4 \%[95 \%$ confidence interval (CI), 24.1\%-30.7\%] and $32.7 \%$ (95\% CI, $28.2 \%-37.2 \%$ ) for men with IPSS $\leq 7$ and those with IPSS $\geq 8$, respectively, and there was no statistically significant difference between groups $(\mathrm{p}=0.120)$. In the analysis of patients categorized by serum PSA levels at the initial population screening, no statistically significant differences were found between groups stratified by IPSS in men with serum PSA levels of 0.0 to $6.0 \mathrm{ng} / \mathrm{mL}$ and 
Table 1. Clinical Characteristics of Men Who Underwent Prostate Biopsy During A Kanazawa Prostate-specific Antigen-based Population Screening Program

\begin{tabular}{|c|c|c|c|}
\hline Variable & $\begin{array}{l}\text { Prostate } \\
\text { Cancer }\end{array}$ & $\begin{array}{c}\text { No Prostate } \\
\text { Cancer }\end{array}$ & P-value \\
\hline Men (n) & 544 & 1195 & \\
\hline \multicolumn{4}{|c|}{ Age at initial screening (years) } \\
\hline Mean \pm SD & $63.4 \pm 4.4$ & $62.6 \pm 4.1$ & $0.0122 *$ \\
\hline Median & 63 & 63 & \\
\hline Range & $54-75$ & $54-75$ & \\
\hline \multicolumn{4}{|c|}{ PSA at initial screening $(\mathrm{ng} / \mathrm{mL})$} \\
\hline Mean \pm SD & $20.9 \pm 169.1$ & $3.9 \pm 4.0$ & $<0.0001 *$ \\
\hline Median & 3.6 & 3 & \\
\hline Range & $0.2-3348.8$ & $0.4-71.1$ & \\
\hline \multicolumn{4}{|l|}{ IPSS at initial screening } \\
\hline Mean \pm SD & $7.3 \pm 6.3$ & $8.0 \pm 6.8$ & $0.1102 *$ \\
\hline Median & 6 & 7 & \\
\hline Range & $0-33$ & $0-35$ & \\
\hline IPSS of 8 or higher $(\mathrm{n}, \%)$ & $216(39.7)$ & $519(43.4)$ & $0.1448 * *$ \\
\hline IPSS of 20 or higher $(\mathrm{n}, \%)$ & $23(4.2)$ & $87(7.3)$ & $0.0153 * *$ \\
\hline \multicolumn{4}{|c|}{ Screening to diagnosis (day) } \\
\hline Mean \pm SD & $856.9 \pm 1079.5$ & $840.8 \pm 1186.8$ & $0.1006 *$ \\
\hline Median & 112.5 & 375 & \\
\hline Range & $6-4725$ & $5-4778$ & \\
\hline \multicolumn{4}{|c|}{ Number of prostate biopsies } \\
\hline Mean \pm SD & $1.2 \pm 0.49$ & $1.2 \pm 0.40$ & $0.6430 *$ \\
\hline Median & 1 & 1 & \\
\hline Range & $1-4$ & $1-6$ & \\
\hline
\end{tabular}

Abbreviation: IPSS, International Prostate Symptom Score *Mann-Whitney U test **Fisher's exact test

Table 2. Population Screening Results for Men Who Underwent Prostate Biopsy and were Stratified by LUTS

\begin{tabular}{llll}
\hline Variable & $\begin{array}{l}\text { Absent or } \\
\text { Mild LUTS } \\
\text { (IPSS of 0-7) }\end{array}$ & $\begin{array}{l}\text { Moderate or } \\
\text { Severe LUTS } \\
\text { (IPSS of 8-35) }\end{array}$ & P-value \\
\hline Number of men & 1004 & 735 & \\
Age at initial screening (years) & $62.4 \pm 4.1$ & $63.6 \pm 4.3$ & $<0.0001^{*}$ \\
Mean \pm SD & 63 & 64 & \\
Median & $54-75$ & $54-73$ & \\
Range & & & \\
Initial PSA (ng/mL) & $6.4 \pm 130.9$ & $13.1 \pm 55.2$ & $<0.0001^{*}$ \\
Mean \pm SD & 2.9 & 3.2 & \\
Median & $0.4-1730.0$ & $0.2-3348.8$ & \\
Range & & & \\
Initial PSA range (ng/mL) & $278(27.7)$ & $122(16.6)$ & $<0.0001^{* *}$ \\
0.0-2.0 (n, \%) & $400(39.8)$ & $299(40.7)$ & $(\leq 2 v s \geq 2.1)$ \\
2.1-4.0 (n, \%) & $248(24.7)$ & $240(32.7)$ & \\
$4.1-10.0(\mathrm{n}, \%)$ & $78(7.8)$ & $73(9.93)$ & \\
$>10.0(\mathrm{n}, \%)$ & $328(32.7)$ & $216(29.4)$ & $0.1448 * *$ \\
Ca patients (n, \%) & & & \\
\hline
\end{tabular}

Abbreviations: LUTS, lower urinary tract symptoms; IPSS, International Prostate Symptom Score*Mann-Whitney U test **Fisher's exact test

those with serum PSA levels of $\geq 12.1 \mathrm{ng} / \mathrm{mL}$ (Figures. 1A, 1B, and 1D). Among men with serum PSA levels of 6.1 to $12.0 \mathrm{ng} / \mathrm{mL}$ at initial screening, the probability of prostate cancer detection was significantly higher in men with IPSS $\leq 7$ than in those with IPSS $\geq 8$ (Figure. 1C).

The clinical characteristics of patients with prostate cancer in the present study are shown in Table 3. Among 544 patients, $125(23.0 \%)$ were diagnosed at serum PSA levels of $\geq 10.1 \mathrm{ng} / \mathrm{mL}$. Fifteen $(2.8 \%)$ had locally advanced cancer and $11(2.0 \%)$ had metastatic disease. There were no significant differences in serum PSA levels at diagnosis, Gleason scores in biopsy specimens, and clinical stage
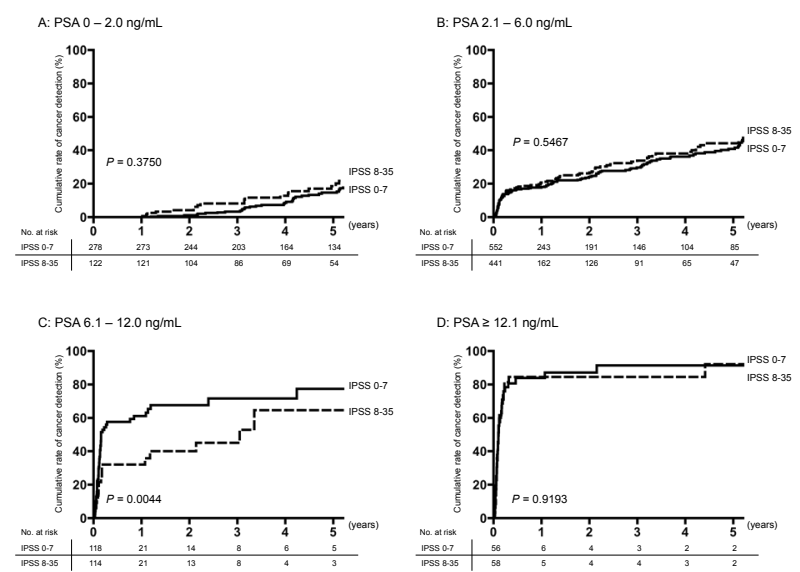

Figure 1. Cumulative Probability of Prostate Cancer Detection Using IPSS During Follow-up in Men with (A) Serum PSA Levels of 0-2.0ng/mL, 2.1-6.0ng/mL, (C) 6.1-12.0ng/mL, and (D) $\geq 12.1 \mathrm{ng} / \mathrm{mL}$

Table 3. Clinical Characteristics of Patients Diagnosed with Prostate Cancer and Stratified by LUTS at Initial Screening During a Kanazawa Prostate-specific Antigen-based Population Screening Program

\begin{tabular}{|c|c|c|c|}
\hline Variables & $\begin{array}{l}\text { Absent or } \\
\text { mild LUTS } \\
\text { (IPSS, 0-7) }\end{array}$ & $\begin{array}{l}\text { Moderate or } \\
\text { severe LUTS } \\
\text { (IPSS, 8-35) }\end{array}$ & P-value \\
\hline Patient No. & 328 & 216 & \\
\hline \multicolumn{4}{|c|}{ PSA levels at diagnosis (ng/mL) } \\
\hline Mean \pm SD & $14.1 \pm 96.3$ & $34.8 \pm 240.4$ & \multirow[t]{3}{*}{$0.2804 *$} \\
\hline Median & 5.1 & 5.2 & \\
\hline Range & $2.1-1730.0$ & $2.1-3348.8$ & \\
\hline \multicolumn{4}{|c|}{ Gleason score $(\mathrm{n}, \%)$} \\
\hline$\leq 6$ & $136(41.5)$ & $96(44.4)$ & \multirow[t]{4}{*}{ NS** } \\
\hline 7 & $109(33.2)$ & $55(25.5)$ & \\
\hline $8-10$ & $54(16.5)$ & $39(18.1)$ & \\
\hline Unknown & $29(8.8)$ & $26(12.0)$ & \\
\hline \multicolumn{4}{|c|}{ Clinical stage $(\mathrm{n}, \%)$} \\
\hline T1c N0 M0 & $185(56.4)$ & $121(56.0)$ & \multirow[t]{5}{*}{ NS** } \\
\hline T2 N0 M0 & $116(35.4)$ & $75(33.8)$ & \\
\hline T3, T4 N0 M0 & $8(2.4)$ & $7(3.2)$ & \\
\hline $\mathrm{N} 1$ or $\mathrm{M} 1$ & $5(1.5)$ & $6(2.8)$ & \\
\hline Unknown & $14(4.3)$ & $7(3.2)$ & \\
\hline
\end{tabular}

between the two groups of patients stratified by IPSS at initial screening.

\section{Discussion}

A large European survey demonstrated that only $1 \%$ of the general population was aware that prostate cancer could be asymptomatic (Schulman et al., 2003). This finding indicates the significance of population-based screening for men with asymptomatic or mild LUTS. Indeed, it was found that asymptomatic patients diagnosed with prostate cancer through PSA testing had a better prognosis compared with symptomatic patients (Kubota et al., 2003; Kitagawa et al., 2013). On the other hand, men with voiding symptoms often have an underlying fear of prostate cancer. Since the 1990's, most clinicians have been conducting PSA measurements in men with a complaint of LUTS, and such testing has proved to be useful for the early detection of prostate cancer (Young et al., 2000; Brown et al., 2003). In the present study, the 
proportion of men with severe LUTS was significantly lower in men detected with prostate cancer during screening than in those without cancer, suggesting the possible existence of men with LUTS who underwent PSA testing outside the population screening program.

Previous studies revealed that benign prostatic hyperplasia (BPH) affected serum PSA levels, and elevated serum PSA levels could be a proxy parameter of prostate volume in patients with BPH (Hochberg et al., 2000; Mochtar et al., 2003; Verim L et al., 2013). Considering this consensus, serum PSA levels may be higher in men with LUTS than in those without LUTS in the entire population, a possibility addressed in several studies (Matsubara et al., 2006; Franlund et al., 2012). Similar results were obtained in our present study, suggesting that high serum PSA levels in men with LUTS is not a predictor of positive prostate biopsies obtained during population screening.

Interestingly, in terms of the relationship between prostate cancer detection and the presence of LUTS, previous studies reported different findings in the population screening cohort. Matsubara et al. demonstrated that the detection rate of prostate cancer was not significantly different when based on the presence of LUTS (Matsubara et al., 2006). In contrast, Franlund et al. demonstrated that the absence of voiding symptoms in men with elevated PSA levels was an independent risk factor for prostate cancer detection. Another Japanese hospital-based cohort study demonstrated that absent or mild LUTS was positively associated with prostate cancer in men with serum PSA $<10 \mathrm{ng} / \mathrm{mL}$ (Ito et al., 2013). This discrepancy in prostate cancer detection according to the presence of LUTS may derive from the different distribution of serum PSA ranges in each study. Using the hypothesis that the relationship between cancer detection and the presence of LUTS fluctuates according to serum PSA levels, we investigated the cumulative probability of cancer detection using IPSS in prostate biopsy samples from patients categorized by serum PSA levels. PSA was significantly higher in men with IPSS $\leq 7$ than in those with IPSS $\geq 8$ among men with serum PSA levels of 6.1 to $12.0 \mathrm{ng} / \mathrm{mL}$ at initial screening. In this range of serum PSA levels, asymptomatic or mild LUTS may be a predictor of future cancer detection in prostate biopsy samples. In addition, men with moderate or severe LUTS may have $\mathrm{BPH}$ with a high prostate volume, which causes elevated serum PSA levels. In men with serum PSA levels below $6.1 \mathrm{ng} / \mathrm{mL}$, it is possible that the absence of LUTS will not allow distinction between prostate cancer and BPH because the prostate volume in men with $\mathrm{BPH}$ is generally too small to cause LUTS. Moreover, a high probability of cancer detection was demonstrated, regardless of the presence of LUTS, in men with serum PSA levels above $12.0 \mathrm{ng} / \mathrm{mL}$. Our results clearly showed the relationship between prostate cancer detection and IPSS in the participants of a PSA-based population screening; to our knowledge, this is the first report to demonstrate this relationship according to serum PSA levels.

With regard to the clinical characteristics of men with prostate cancer detected during screening, there was no significant difference in serum PSA levels at diagnosis,
Gleason scores in biopsy specimens, and clinical stage according to LUTS status in the present study. Considering that the proportion of patients with advanced disease was low (locally advanced cancer, $2.8 \%$; metastatic disease, $2.0 \%$ ), men with a high IPSS associated with prostate cancer seemed to be rare in our population-based screening cohort. This finding supported the fact that absent or mild LUTS in men with elevated serum PSA levels constituted a useful predictor of prostate cancer detection during population-based screening.

Considering the retrospective nature of the analysis in the screening cohort, this study had several limitations. First, there were no data regarding prostate volume and PSA density (PSAD) for the screening participants; therefore, the relationship between prostate volume and IPSS could not be demonstrated. It is clear that prostate volume primarily affects LUTS status and that prostate volume should be demonstrated for more valuable evidence. PSAD has been demonstrated to improve specificity (Zheng XY et al., 2012); however, a meaningful interpretation has yet to be uniformly accepted within clinical practice (Gretzer et al., 2003). Further large-scale prospective studies including PSAD data will eventually reveal the optimal threshold of PSAD in prostate biopsies from patients categorized by serum PSA levels. Second, there was a possible bias caused by the fact that participants with LUTS were more likely to undergo prostate biopsies compared with those without. Despite these concerns, our finding that asymptomatic or mild LUTS may be a reliable predictor of future cancer detection in prostate biopsy samples from men with serum PSA levels of 6.1 to $12.0 \mathrm{ng} / \mathrm{mL}$ is very informative for primary care physicians who have a role to interpret screening results (Kwon HT et al., 2013), considering that serum PSA levels and IPSS are obtained through population-based screening alone. In the clinical setting, physicians can use IPSS, which reflects the presence of LUTS, as a reference point for deciding if prostate biopsy is warranted in men with this range of serum PSA levels.

\section{Acknowledgements}

We thank Dr. Yasuo Takeda and Dr. Atsushi Hashiba of Kanazawa Medical Association for providing data on the Kanazawa population-based screening cohort.

\section{References}

Bouchardy C, Fioretta G, Rapiti E, et al (2008). Recent trends in prostate cancer mortality show a continuous decrease in several countries. Int J Cancer, 123, 421-9.

Brown CT, O'Flynn E, van der Meulen J, et al (2003). The fear of prostate cancer in men with lower urinary tract symptoms: should symptomatic men be screened? BJU Int, 91, 30-2.

Franlund M, Carlsson S, Stranne J, Aus G, Hugosson J (2012). The absence of voiding symptoms in men with a prostatespecific antigen (PSA) concentration of $\geq 3.0 \mathrm{ng} / \mathrm{mL}$ is an independent risk factor for prostate cancer: results from the Gothenburg randomized screening trial. BJU Int, 110, 638-43.

Gretzer MB, Partin AW (2003). PSA markers in prostate cancer detection. Urol Clin North Am, 30, 677-86.

Hochberg DA, Armenakas NA, Fracchia JA (2000). Relationship 
of prostate-specific antigen and prostate volume in patients with biopsy proven benign prostatic hyperplasia. Prostate, 45, 315-9.

International Union Against Cancer (1997). Urologic Tumors: Prostate. In 'TNM Classification of Malignant Tumours. 5th ed.' Eds Sobin LH and Wittekind CH. John Wiley \& Sons, New York pp170-3

Ito K (2009). Prostate-specific antigen-based screening for prostate cancer: evidence, controversies and future perspectives. Int J Urol, 16, 458-64.

Ito M, Masuda H, Kawakami S, et al (2013). Impact of lower urinary tract symptoms on prostate cancer risk among Japanese men with prostate-specific antigen $<10 \mathrm{ng} / \mathrm{ml}$ and non-suspicious digital rectal examination. Int $J$ Urol, 20, 1163-8.

Kash DP, Lal M, Hashmi H, Mubarak M (2014). Utility of digital rectal examination, serum prostate specific antigen, and transrectal ultrasound in the detection of prostate cancer: a developing country perspective. Asian Pac J Cancer Prev, 15, 3087-91.

Kitagawa Y, Mizokami A, Nakashima K, et al (2011). Clinical outcomes of prostate cancer patients detected by prostatespecific antigen-based population screening in Kanazawa city, Japan. Int J Urol, 18, 592-6.

Kitagawa Y, Mizokami A, Namiki M (2013). Trends of clinical symptoms and prognosis of middle-aged prostate cancer patients after instigation of prostate specific antigen-based population screening. Prostate Int, 1, 65-8.

Kobori Y, Kitagawa Y, Mizokami A, et al (2008). Free-to-total prostate-specific antigen (PSA) ratio contributes to an increased rate of prostate cancer detection in a Japanese population screened using a PSA level of $2.1-10.0 \mathrm{ng} / \mathrm{ml}$ as a criterion. Int J Clin Oncol, 13, 229-32.

Kubota Y, Ito K, Imai K, et al (2002). Effectiveness of mass screening for the prognosis of prostate cancer patients in Japanese communities. Prostate, 50, 262-9.

Kwon HT, Ma GX, Gold RS, Atkinson NL, Wang MQ (2013). Primary care physician's cancer screening recommendation practices and perceptions of cancer risk of Asian Americans. Asian Pac J Cancer Prev, 14, 1999-2004.

Matsubara A, Yasumoto H, Teishima J, et al (2006). Lower urinary tract symptoms and risk of prostate cancer in Japanese men. Int J Urol, 13, 1098-102.

Mochtar CA, Kiemeney LA, van Riemsdijk MM, et al (2003). Prostate-specific antigen as estimator of prostate volume in the management of patients with symptomatic benign prostatic hyperplasia. Eur Urol, 44, 695-700.

Okihara K, Kitamura K, Okada K, et al (2008). Ten year trend in prostate cancer screening with high prostate-specific antigen exposure rate in Japan. Int J Urol, 15, 156-61.

Schulman CC, Kirby R, Fitzpatrick JM (2003). Awareness of prostate cancer among the general public: findings of an independent international survey. Eur Urol, 44, 294-302.

Shin S, Kim YH, Hwang JS, et al (2014). Economic evaluation of prostate cancer screening test as a national cancer screening program in South Korea. Asian Pac J Cancer Prev, 15, 3383-9.

Verim L, Yildirim A, Basok EK, et al (2013). Impact of PSA and DRE on histologic findings at prostate biopsy in Turkish men over 75 years of age. Asian Pac J Cancer Prev, 14, 6085-8.

Young JM, Muscatello DJ, Ward JE (2000). Are men with lower urinary tract symptoms at increased risk of prostate cancer? A systematic review and critique of the available evidence. BJU Int, 85, 1037-48.

Zheng XY, Zhang P, Xie LP, et al (2012). Prostate-specific antigen velocity (PSAV) and PSAV per initial volume (PSADV) for early detection of prostate cancer in Chinese men. Asian Pac J Cancer Prev, 13, 5529-33. 